\title{
SEPT12 Interacts with SEPT6 and This Interaction Alters the Filament Structure of SEPT6 in Hela Cells
}

\author{
Xiangming Ding, Wenbo Yu, Ming Liu, Suqin Shen, Fang Chen, Bo Wan and Long Yu* \\ State Key Laboratory of Genetic Engineering, Institute of Genetics, School of Life Science, Fudan University, Shanghai 200433, PR China
}

Received 5 June 2007, Accepted 25 July 2007

\begin{abstract}
Septins are a family of conserved cytoskeletal GTPase forming heteropolymeric filamentous structure in interphase cells, however, the mechanism of assembly are largely unknown. Here we described the characterization of SEPT12, sharing closest homology to SEPT3 and SEPT9. It was revealed that subcelluar localization of SEPT12 varied at interphase and mitotic phase. While SEPT12 formed filamentous structures at interphase, it was localized to the central spindle and to midbody during anaphase and cytokinesis, respectively. In addition, we found that SEPT12 can interact with SEPT6 in vitro and in vivo, and this interaction was independent of the coiled coil domain of SEPT6. Further, co-expression of SEPT12 altered the filamentous structure of SEPT6 in Hela cells. Therefore, our result showed that the interaction between different septins may affect the septin filament structure.
\end{abstract}

Keywords: Co-localization, Filament structure, Protein interaction, Septin, Subcellular localization

\section{Introduction}

Septins are an evolutionarily conserved family of polymerizing GTP binding proteins originally discovered in budding yeast as a group of cell cycle mutants which cause defects in cytokinesis (Hartwell, 1971; Kinoshita, 2003). It is now clear that septins are present in the fungi and animals, although apparently not in plants (Field and Kellogg, 1999). All septins contain a conserved GTPase domain in the center and most septins have divergent $\mathrm{N}$ - and $\mathrm{C}$-terminal domains, many of which contain a predicted coiled-coil region possibly involved in protein-protein interaction. In addition to their widespread involvement in cytokinesis (Kinoshita et al.,

\footnotetext{
*To whom correspondence should be addressed.

Tel: 86-21-65643717; Fax: 86-21-65643250

E-mail: longyu@fudan.edu.cn
}

1997; Surka et al., 2002), septins are implicated in a variety of other celluar processes including polarity determination (Gladfelter et al., 2001; Irazoqui et al., 2004), vesicle trafficking (Hsu et al., 1998; Beites et al., 1999), cytoskeletal remodelling (Kinoshita et al., 1997; Surka et al., 2002) ,apoptosis (Larisch et al., 2000) and neoplasia (Russel and Hall, 2005).

Up to date, seven yeast septins have been identified: cdc3, cdc10, cdc11, cdc12, SPR3, SPR28, and SEP7/SHS1 (DeVirgilio et al., 1996; Mino et al., 1998) and at least five septins, namely PNUT1, SEP1, SEP2, SEP4 and SEP5, are known in Drosophila (Adam et al., 2000). As for mammalian cells, 13 septin genes have so far been identified mainly based on random sequencing projects (Hall et al., 2005) and most of them have been characterized. Many mammalian septin genes are likely to undergo complex splicing, giving rise to an even greater number of distinct transcripts, and hence septin protein isoforms in specific cell types.

The accumulating biochemical and cell biological observations on lower eukaryotic septins suggest that septins form filament structures, which are most likely to be essential to their function (Versele et al., 2005). However, little is known about how these filaments were formed and how the filament formation was regulated. A recent study showed that Borg3, a downstream effector of the Cdc42 GTPase, can markedly alter the filament structures of septins and $\mathrm{Cdc} 42$ negatively regulates this effect by inhibiting the binding of Borg3 to septins (Joberty et al., 2001). Interestingly, Borg3 binds specifically to a septin heterodimer composed of SEPT6 and SEPT7 and to the SEPT2/6/7 trimer, but not to septin monomers (Sheffield et al., 2003). In mammalian cells, two complexes (SEPT2/6/7 and SEPT7/9b/11) have been affinitypurified from Hela cells and REF52 cells, respectively, with anti-SEPT2 and anti-SEPT7 antibody (Kinoshita et al., 2002; Nagata et al.,2004). The multiplicity of mammalian septin genes and their complex splicing patterns suggest that septin complexes with a different composition from the above mentioned are likely to exist.

Although more and more studies have focused on function 
of the mamalian septins for their involvement in a variety of cellular process, little information about SEPT12 has been reported. Here we cloned SEPT12 from testis cDNA library. While SEPT12 was present in filamentous structure at cytoplasm in interphase, it transferred to the central spindle and midbody during anaphase and cytokinesis, respectively. In addition, since septins always form heteropolymers with other septins, we deduced that SEPT12 may interact with other septins. Consequently, we examined the possible interaction of SEPT12 with other septins in our lab and found SEPT12 can interact with SEPT6 and SEPT11. In this study we focused on the interaction between SEPT12 and SEPT6. We found that SEPT12 can interact with SEPT6 in vitro and in vivo and this interaction changed the filamentous structure of SEPT6 in Hela cells.

\section{Materials and Methods}

Molecular cloning and bioinformatic analysis. PCR primers (forward primer: 5'-TCCACCAGGCATCTCGAACCCTTG-3'; reverse primer: 5'-CGGTGGTCAGAACTCATCATCAG-3') were designed and synthesized according to the SEPT12 cDNA sequence (GenBank accession number: NM_144605). Human testis cDNA library was used as a template, and the PCR amplification was performed by using the conditions as $95^{\circ} \mathrm{C}$ for $5 \mathrm{~min}$ followed by 32 cycles of $95^{\circ} \mathrm{C}$ for $30 \mathrm{~s}, 56^{\circ} \mathrm{C}$ for $45 \mathrm{~s}$ and $72^{\circ} \mathrm{C}$ for $60 \mathrm{~s}$. The PCR product was suncloned into pMD18-T vector (TaKaRa) and subjected to sequencing. Protein sequences were aligned by Clustal $\mathrm{X}$ software and viewed by GENEDOC software. Phylogenetic tree was constructed using the Neighbor-Joining (N-J) method implemented with PHYLIP software (Felsenstein., 1989) Bootstrap analysis with 1000 replicates was performed to assess relative confidence in the topologies obtained.

Plasmid construction. For the bacterial expression of the fusion protein, the SEPT12 cDNA was subcloned in frame with GST tag into the pGEX-4T-1 (Amersham Biosciences). To investigate subcellular localization, human SEPT12 and SEPT6 (a gift kindly from Dr Jim Crawford, University of Virginia) cDNAs were introduced into the pFLAG-CMV2 (Clontech) and pCMV-myc (Clontech) respectively. In addition, the cDNA fragment of SEPT6N (amino acid: 1-320), with a deletion of the coiled coil region, was obtained using PCR and then subcloned into the pCMV-myc. All constructs were verified by DNA sequencing.

Preparation of recombinant proteins. The recombinant glutathione S-transferase (GST) tagged protein was expressed in Escherichia coli strain BL21-CodonPlus(DE3)-RIL with $0.2 \mathrm{mM}$ isopropyl- $\beta$ D-thiogalactopyranoside (IPTG) at $25^{\circ} \mathrm{C}$ overnight. Cells were collected and lysed in the buffer of PBS ( $\mathrm{pH} 7.0$ ), supplemented with $1 \%$ Triton X-100, $10 \mathrm{mM} \beta$-mercaptoethanol, $0.5 \mathrm{mM}$ PMSF and $1 \mathrm{mg} / \mathrm{ml}$ lysozyme. After incubation on ice for $30 \mathrm{~min}$, the samples were centrifuged and the supernatants were purified using glutathione-S-Sepharose beads (Amersham Biosciences) as recommended by the manufacturers.
Cell culture and transfection. Hela cells were cultivated in Dulbecco's modified Eagle's medium (GIBCO) supplemented with $10 \%$ fetal calf serum (Invitrogen, USA) at $37^{\circ} \mathrm{C}$ in $5 \% \mathrm{CO}_{2}-$ humidified atmosphere. $1.2 \times 10^{5}$ cells were seeded on coverslips in $35 \mathrm{~mm}$ dishes. After overnight growth cells were $80 \%$ confluent and transfected with $2 \mu \mathrm{g}$ of plasmid constructs using Lipofectamine ${ }^{\mathrm{TM}}$ Reagent (Invitrogen) in the non-serum medium. After $5 \mathrm{~h}$ of incubation medium was replaced with fresh complete medium, and cells were cultured for an additional $30 \mathrm{~h}$ before collection.

GST pull-down assays. $10^{6}$ transfected Hela cells were harvested and lysed in $0.5 \mathrm{ml}$ of cell lysis buffer $(20 \mathrm{mM}$ Tris- $\mathrm{HCl}, \mathrm{pH} 7.5$, $150 \mathrm{mM} \mathrm{NaCl}, 1 \mathrm{mM} \mathrm{Na}{ }_{2}$ EDTA, $1 \mathrm{mM}$ EGTA, 1\% (v/v) Triton X$100,2.5 \mathrm{mM}$ sodium pyrophosphate, $1 \mathrm{mM} \beta$-glycerophosphate, $1 \mathrm{mM} \mathrm{Na} 3 \mathrm{VO} 4,1 \mu \mathrm{g} / \mathrm{mL}$ leupeptin). Binding assays were initiated by incubating cell extracts in lysis buffer with $5 \mu \mathrm{g}$ GST-SEPT 12 or $5 \mu \mathrm{g}$ GST protein bound to $40 \mu \mathrm{l}$ glutathione-S-Sepharose beads for $5 \mathrm{~h}$ at $4^{\circ} \mathrm{C}$. The beads were washed five times with lysis buffer and analyzed by Western blotting.

Co-immunoprecipitation. Cells were harvested and lysed described in GST pull-down assay. The lysates were preclarified with protein A/G Plus-agarose by rotating at $4^{\circ} \mathrm{C}$ for $30 \mathrm{~min}$. After the beads were discarded by centrifugation, the lysates were then immunoprecipitated with primary antibody bound to protein A/G-Sepharose beads for $8 \mathrm{~h}$ at $4^{\circ} \mathrm{C}$. The beads were washed five times with cell lysis buffer and finally analyzed by Western blotting.

Western blotting. Samples were resolved by $12 \%$ SDS-PAGE and then transferred to nitrocellulose memberanes, and these were separately incubated with antibodies against the Flag-tag (M2 monoclonal antibody, Sigma), myc-tag (c-myc monoclonal antibody, BD Biosciences), and GST-tag (GST-tag monoclonal antibody, Novagen), followed by horseradish peroxidase-conjugated rabbit anti-mouse antibodies, respectively. Immunoreactivity was visualized by enhanced chemiluminescence.

Immunofluorescence microscopy. Cells grown on coverslips were fixed in preiced $3.7 \%(\mathrm{w} / \mathrm{v})$ formaldehyde/PBS $(\mathrm{pH} 7.0)$ for 10 min. After being washed with PBS three times, cells were resolved by $0.2 \%(\mathrm{v} / \mathrm{v})$ Triton $\mathrm{X}-100$ for $15 \mathrm{~min}$ at room temperature. and then the permeabilized cells were blocked by a solution containing $10 \%(\mathrm{v} / \mathrm{v})$ horse serum and $1 \%(\mathrm{w} / \mathrm{v})$ BSA in PBS for $1 \mathrm{~h}$ at room temperature. The diluted primary antibodies including antibodies against the Flag-tag (M2 monoclonal antibody or pAb polyclonal antibody, Sigma) or/and myc-tag (c-myc monoclonal antibody, BD Biosciences) were placed as a drop on the coverslips and incubated for $2 \mathrm{~h}$ at room temperture in a humidified chamber. The cells were then washed and covered with Cy3-conjugated donkey anti-mouse (Jackson ImmunoResearch) or/and Alex488-conjugated goat antirabbit antibody (Molecular Probes), respectively, for $1 \mathrm{~h}$ in the dark. In some experiments, nuclei were stained with 4,6-diamidino-2phenylindole (DAPI). Fluorescence was analysed using a Leica DMRA2 microscope or a Leica TCS-NT laser confocal microscope. 


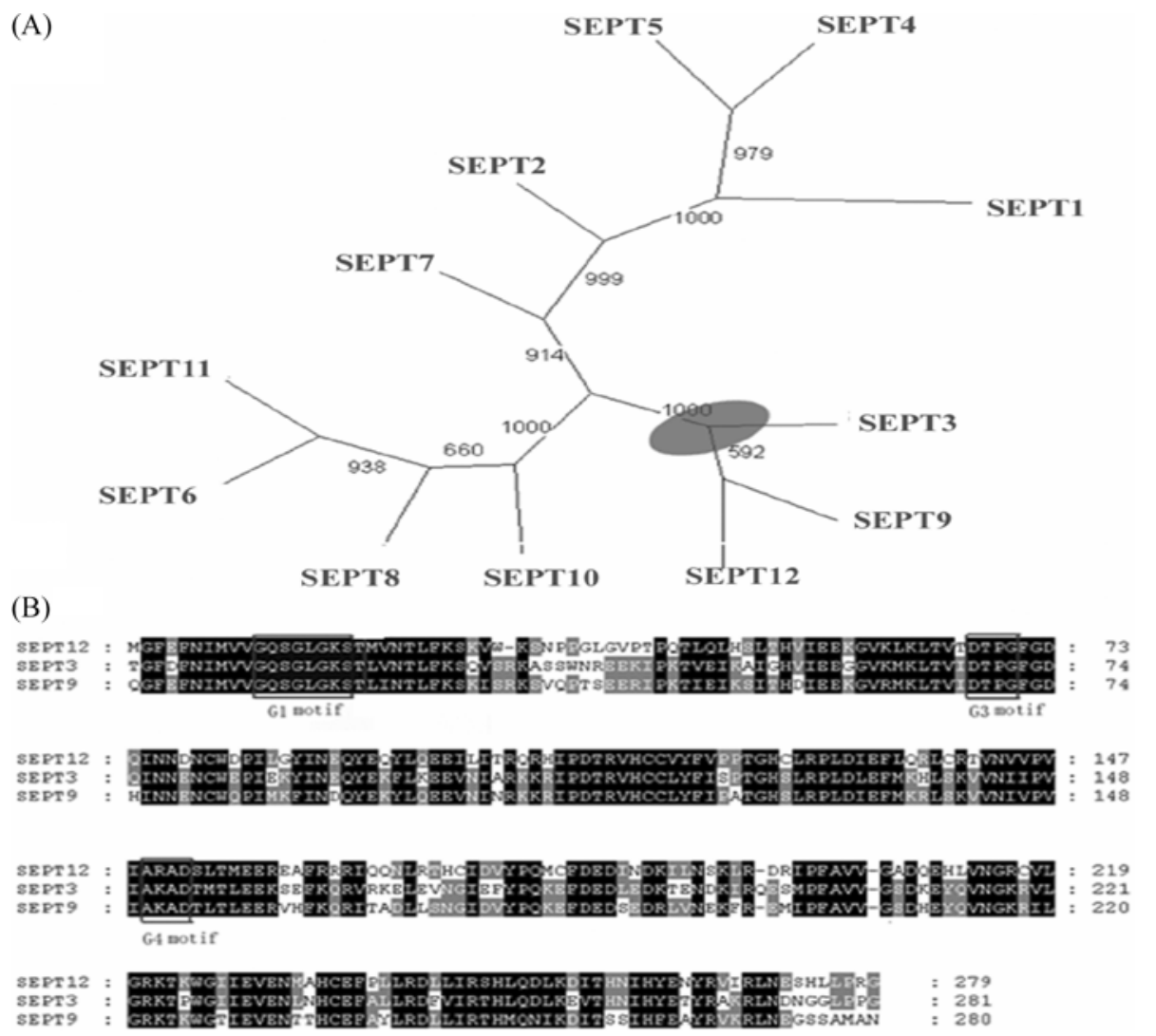

Fig. 1. (A) Phylogenetic tree of the human septin family members. The unrooted phylogenrtic tree was constructed using the NeighborJoining (N-J) method implemented with PHYLIP software. Bootstrap values are shown at each node. (B) Alignment of the GTPase domain in SEPT12, SEPT3 and SEPT9. Identity is indicated by black shading, and the similarity is indicated by gray shading. Conserved amino acid substitutions are defined as pairs of residues belonging to one of the following groups: S/T/P/A/G; N/D/E/Q; H/R/K; M/I/L/V and F/Y/W. G1 motif, G2 motif and G3 motif are boxed.

\section{Results}

Sequence analysis of human SEPT12. Result of searching in SMART database (http://smart.emblheidelbeg.de) showed SEPT12 protein contains a central GTPase domain, which is highly conserved in septin family and generally regarded to be essential for their functions. On the basis of the GTPase domains, human septin proteins were phylogenetically analyzed using the Neighbor-Joining (N-J) method implemented with PHYLIP software. As shown in Fig. 1A, SEPT12 is most closely related to SEPT3 and SEPT9, exhibiting 61-63\% identity and $76-77 \%$ similarity (Fig. 1B).

Subcellular characterization of SEPT12 protein. To investigate the subcellular localization of SEPT12, Flagtagged SEPT12 construct was transfected into Hela cells and $36 \mathrm{~h}$ after the transfection, cells were processed with DAPI and anti-Flag M2 antibody for the localization of SEPT12. We found that the localization of SEPT12 was variable at different cell cycle stages (Fig. 2). SEPT12 was present around the nucleus and appeared as filamentous structures in interphase. The filamentous appearance of SEPT12 disappeared at the

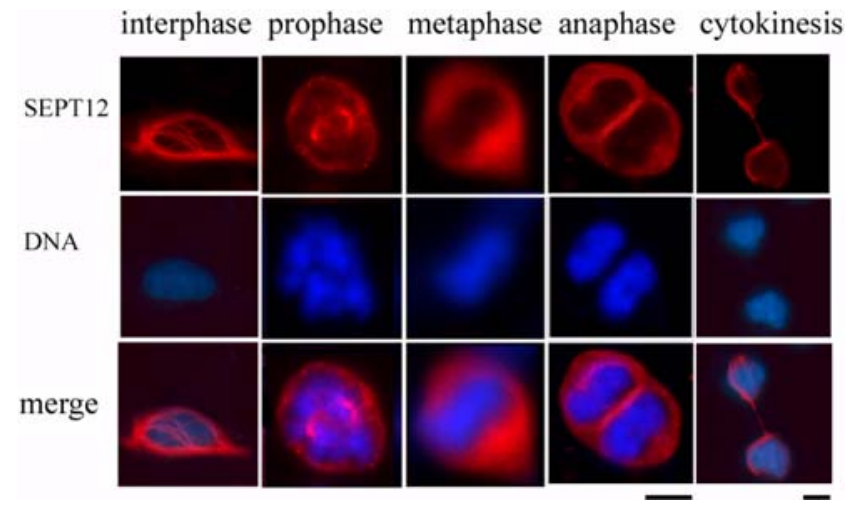

Fig. 2. Subcellular localization of SEPT12 in interphase and mitotic phase. Cells were transiently transfected with Flag-tagged SEPT12 construct for $36 \mathrm{~h}$, fixed, and subjected to anti Flag immunostaining (red). Cells were counterstained with DAPI (blue). The fluorescence was analysed using a Leica DMRA2 microscope. The scale bar reprents $10 \mu \mathrm{m}$.

onset of mitosis. During anaphase, SEPT12 exhibited a punctuate staining at the central spindle. After the formation 
(A)

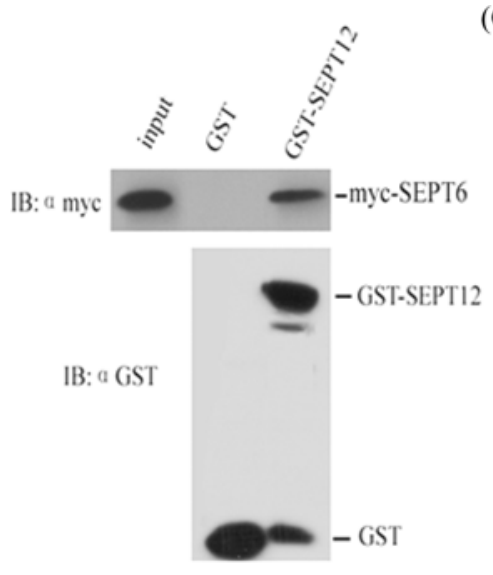

(C)

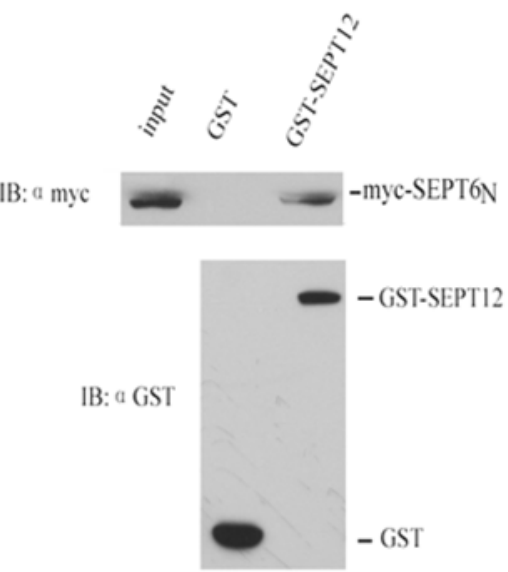

(B)

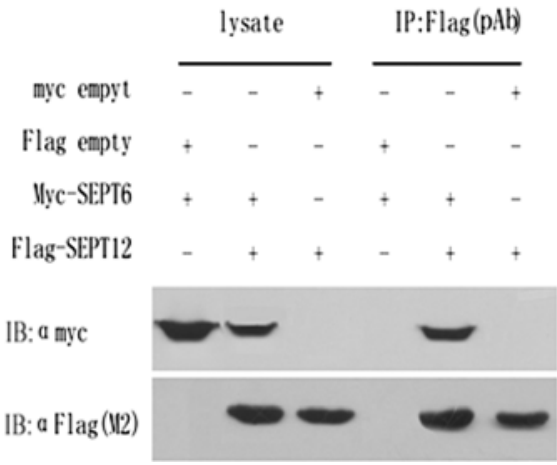

Fig. 3. Interaction of SEPT12 and SEPT6 in vitro and in vivo (A) GST-SEPT12 pull-down myc-SEPT6. Lysates prepared from Hela cells expressing myc-SEPT6 were incubated with GST alone or GST-SEPT12 that prebound to glutathione beads. Bound myc-SEPT6 was detected by immunoblot with anti-myc antibody. (B) Co-immunoprecipiation of Flag-SEPT12 and myc-SEPT6. Flag-SEPT12 and mycSEPT6 were transiently expressed either with myc and Flag-tag, respectively, or expressed together in Hela cells. Transfection conditions for each lane are indicated at the top of the panels. Total cellular lysates and Flag-SEPT12 immunopercipiates (IP: $\alpha$ Flag pAb) were probed with anti-Flag (M2) antibody and anti-myc monoclonal antibodies. (C) GST-SEPT12 pull-down myc-SEPT6N. Lysates prepared from Hela cells expressing myc-SEPT6N were incubated with GST alone or GST-SEPT12 that prebound to glutathione beads. Bound myc-SEPT6N was detected by immunoblot with anti-myc antibody.

of the midbody in cytokinesis, SEPT12 was localized to the midbody.

SEPT12 binds to SEPT6 in vitro and in vivo. The interaction between SEPT12 and SEPT6 proteins was first examined using in vitro GST pull-down assay. SEPT12 was expressed as a GST fusion (GST-SEPT12), and both this fusion product and GST alone were purified using glutathioneS-Sepharose beads. Lysates were prepared from Hela cells transfected with the plasmid pCMV-myc-SEPT6. This lysate was then incubated with glutathione beads complexed with either GST alone or with GST-SEPT12. Beads were then washed and bound proteins were separated by SDS-PAGE. SEPT6 was detected by Western blot using anti-myc antibody. The results show that SEPT6 protein interacted with GSTSEPT12 but not GST alone (Fig. 3A).

The result from the pull-down experiments suggested that SEPT12 and SEPT6 proteins can bind in vitro. To further characterize the in vivo interaction of SEPT12 and SEPT6, co- immunoprecipitation was performed on Hela cells. Hela cells were transiently transfected with Flag-tagged SEPT12 and myc-tagged SEPT6. The total lysates were subjected to immunoprecipitation using the anti-Flag pAb polyclonal antibody, and immunoblots containing protein complexes were probed with the anti-Flag M2 antibody and anti-myc monoclonal antibody. As shown in Fig. 3B, Myc-SEPT6 was co-immunoprecipitated with Flag-SEPT12 but not with others. Taken together, these results provided evidences that SEPT12 and SEPT6 can interact both in vitro and $i v$ vivo.

In addition, previous study showed that in some cases the coiled coil domain of septin is implicated in the septin-septin interaction (Sheffield et al., 2003). However, SEPT12 lacks such a domain, so we examined whether the coiled coil domain of SEPT6 was required for this interaction. GST pulldown assay showed that SEPT 12 can interact with SEPT@N (Fig. 3C), indicating that this interaction was independent of the coiled coil domain of SEPT6. 
(A)
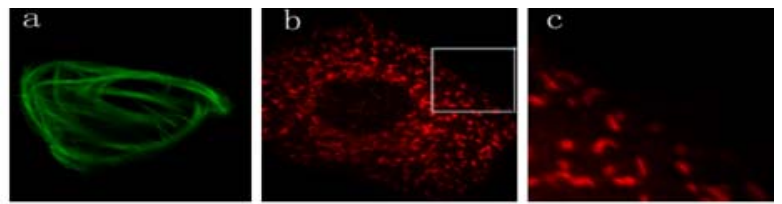

(B)
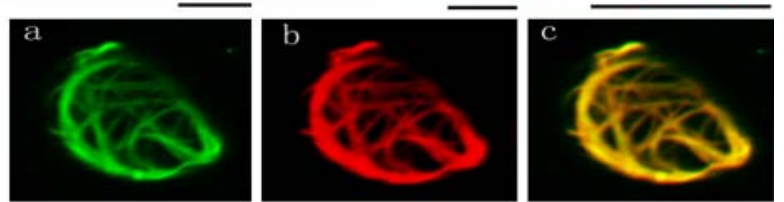

(C)
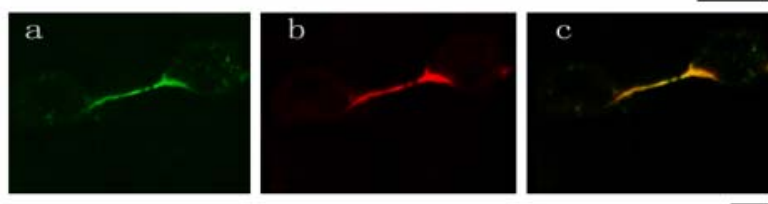

Fig. 4. Co-expressiopn of SEPT12 affects the SEPT6 filament structures. (A) Flag-SEPT12 (a) or myc-SEPT6 (b) was transiently expressed in Hela cells. After $30 \mathrm{~h}$ transfection, cells were fixed and stained with anti-Flag pAb/Alex488-conjugated anti-rabbit antibody or anti myc $\mathrm{mAb} / \mathrm{Cy} 3$-conjugated anti-mouse antibody. Enlarged image of the boxed area of $b$ was shown (c). (B) Flag-SEPT12 and myc-SEPT6 were transiently co-expressed in Hela cells. After $30 \mathrm{~h}$ transfection, cells were fixed and were double-stained with anti-Flag pAb/Alex488-conjugated anti-rabbit antibody (a) and anti myc mAb/ Cy3-conjugated anti-mouse antibody (b). The merge image was shown (c). (C) Hela cells expressing Flag-SEPT12 and myc-SEPT6 during cytokinesis were double-stained as in $\mathrm{B}$. The merge image was shown (c). The fluorescence was analysed using a Leica TCSNT laser confocal microscope. The scale bar reprents $10 \mu \mathrm{m}$.

\section{Co-expression of SEPT12 affects the SEPT6 filament in} Hela cells. Since SEPT12 and SEPT6 can form an in vivo immunocomplex when exogenously expressed in Hela cells, it is likely that the co-expressed septins are also co-localized in Hela cells. We thus test this possiblity. Firstly, the localization pattern of SEPT12 and SEPT6 in Hela cells was examined respectively. We found that SEPT12 per se formed long filaments in Hela cells (Fig. 4A (a)). However the filaments SEPT6 per se formed were much shorter and appeared curling (Fig. 4A (b-c)). Then we explored filament structures when SEPT12 and SEPT6 were co-expressed in Hela cells. We found that compared with filaments formed by SEPT6 per se, the filaments of SEPT6 appeared much longer and became straight (Fig. 4B (b)), when co-expressed with SEPT12, which indicated that co-expression of SEPT12 altered the filamentous structure of SEPT6. On the other hand, co-expression of SEPT6 did not change obviously the filament structure of SEPT12 (Fig. 4A (a) and Fig. 4B (a)). Although the septin filaments observed here did not necessarily reflect their physiological intracellular distribution, effects of a septin on another septin structure was examined. Finally, as shown in Fig. 4B (c) and Fig. 4C (c), SEPT12 and SEPT6 colocalized both in intrerphase and during cytokinesis when coexpressed in Hela cells.

\section{Discussion}

Mammalian septins are expressed widely in human tissues and most septins including SEPT12 appear ubiquitous (Hall et al., 2005). However SEPT12 appears to be expressed at high level in the testis, which is different from other septins (Hall et al., 2005). Septin genes typically undergo complex splicing and SEPT9 has 18 different splice variants, encoding at least 15 different proteins (Mcllhatton et al., 2001). When we amplified SEPT12 from the testis cDNA library, we also found a novel SEPT12 transcript and the GenBank accession number is DQ456996.

Septins may function as filamentous scaffolds in the cytoplasm for organization of proteins in a specific region inside the cell, because many proteins depend on septins for localization (Cooper and Kiehart, 1996; Kinoshita, 2003). However, ARTS, one of the SEPT4 splice variants localized to mitochondria in living cells, is translocated to the nucleus when apoptosis occurs (Larisch et al., 2000). Interestingly, SEPT2 and SEPT9, both known to be required for cytokinesis during mitosis, are found in two distinct locations: SEPT2 is localized mainly to the contractile actomyosin ring, while SEPT9 is found predominantly on the central spindle region in mitotic phase (Kinoshita et al., 1997; Surka et al., 2002). This study showed that SEPT12 displayed obvious filamentous structure in the cytoplasm in interphase and translocated to the central spindle region in anaphase and to the midbody in cytokinesis. Our results demonstrated that the localization feature of SEPT12 is similar to SEPT9, but not to SEPT2.

Despite the recent progress on the mammalian septin complex structure, much less information is available regarding function of mammalian septins as compared with lower eukaryotic cells. In this study we found that SEPT12 can interact with SEPT6 in vitro and in vivo, and this interaction altered the filament structure of SEPT6. Furthermore, we found that SEPT12 can associate with SEPT6 independently of the coiled coil region of SEPT6. Our result is consistent with a recent study which showed that CDC10, a yeast septin lacking coiled coil domain, interacts with a $\mathrm{Cdc} 3-\mathrm{Cdc} 12$ complex independently of the coiled coil domain of either protein (Versele et al., 2004). Until now how the filamentous structures of septins formed was yet largely unclear. A recent study demonstrated that although a purified recombinant Cdc3-Cdc12-Cdc11 complex polymerizes in vitro, the resulting filaments are unstable, short and display marked curvature. By contrast, filaments generated from a purified Cdc3-Cdc12Cdc11-Cdc10 complex are more stable, much longer and straighter, which indicated that $\mathrm{Cdc} 10$ made crucial contributions to septin-filament organization and stability in budding yeast (Versele et al., 2004). In our study human SEPT12, a mammalian counterpart of yeast $\mathrm{Cdc10}$ (Versele and Thorner, 2005), was found to make the filaments formed by SEPT6 much longer at least in Hela cells. Recently, K. Nagata et al. (2004) have showed that SEPT9b, another mammalian counterpart of yeast Cdc10, can make the SEPT7 filaments 
longer and the SEPT11 filaments thicker in COS7 cells. Although further investigation was required, it was tempting to speculate that the SEPT12, SEPT9 and SEPT3, as a septin subgroup, may play a key role in the filament organization of the mammalian septins.

Further studies on the cytologicial and biochemical properties of the mammalian septin complexes were required to clarify the detailed mechanism how the septin-septin interaction influences the filament formation of septins.

Acknowledgments This work was supported by the National 973 Program and 863 High Technology Program of China, as well as the National Natural Science Foundation of China.

\section{References}

Adam, J. C., Pringle, J. R. and Peifer, M. (2000) Evidence for functional differentiation among Drosophila septins in cytokinesis and cellularization. Mol. Biol. Cell 11, 3123-3135.

Beites, C. L., Xie, H., Bowser, R. and Trimble, W. S. (1999) The septin CDCrel-1 binds syntaxin and inhibits exocytosis. Nat. Neurosci. 2, 434-439.

Cooper, J. A. and Kiehart, D. P. (1996) Septins may form a ubiquitous family of cytoskeletal filaments. J. Cell Biol. 134, 1345-1348.

DeVirgilio, C., DeMarini, D. J. and Pringle, J. R. (1996) SPR28, a sixth member of the septin gene family in Saccharomyces cerevisiae that is expressed specifically in sporulating cells. Microbiology 142, 2897-2905.

Felsenstein, J. (1989) PHYLIP-Phylogeny inference package (version 3.2), Cladistics 5, 164-166.

Field, C. M. and Kellogg, D. (1999) Septins: cytoskeletal polymers or signalling GTPases? Trends Cell Biol. 9, 387-394.

Gladfelter, A. S., Pringle, J. R. and Lew, D. J. (2001) The septin cortex at the yeast mother-bud neck. Curr. Opin. Microbiol. 4, 681-689.

Hall, P. A., Jung K., Hillan, K. J. and Russell, S. E. (2005) Expression profiling the human septin gene family. J. Pathol. 206, 269-278.

Hartwell, L. H. (1971) Genetic control of the cell division cycle in yeast. IV. Genes controlling bud emergence and cytokinesis. Exp. Cell Res. 69, 265-276.

Hsu, S. C., Hazuka, C. D., Roth, R., Foletti, D. L., Heuser, J. and Scheller, R. H. (1998) Subunit composition, protein interactions, and structures of the mammalian brain sec6/8 complex and septin filaments. Neuron 20, 1111-1122

Irazoqui, J. E. and Lew, D. J. (2004) Polarity establishment in yeast.
J. Cell Sci. 117, 2169-2171.

Joberty, G., Perlungher, R. R., Sheffield, P. J., Kinoshita, M., Noda, M., Haystead, T. and Macara, I. G. (2001) Borg proteins control septin organization and are negatively regulated by $\mathrm{Cdc} 42$. Nat. Cell Biol. 3, 861-866.

Kinoshita, M. (2003) Assembly of mammalian septins. J. Biochem. (Tokyo) 134, 491-496.

Kinoshita, M., Field, C. M., Coughlin, M. L., Straight, A. F. and Mitchison, T. J. (2002) Self- and actin-templated assembly of Mammalian septins. Dev. Cell 3, 791-802.

Kinoshita, M., Kumar, S., Mizoguchi, A., Ide, C., Kinoshita, A., Haraguchi, T., Hiraoka, Y. and Noda, M. (1997) Nedd5, a mammalian septin, is a novel cytoskeletal component interacting with actin-based structures. Genes Dev. 11, 1535-1547.

Larisch, S., Yi, Y., Lotan, R., Kerner, H., Eimerl, S., Tony, P. W., Gottfried, Y., Birkey, R. S., deCaestecker, M. P., Danielpour, D., Book-Melamed, N., Timberg, R., Duckett, C. S., Lechleider, R. J., Steller, H., Orly, J., Kim, S. J. and Roberts, A. B. (2000) A novel mitochondrial septin-like protein, ARTS, mediates apoptosis dependent on its P-loop motif. Nat. Cell Biol. 2, 915-921.

McIlhatton, M. A., Burrows, J. F., Donaghy, P. G., Chanduloy, S., Johnston, P. G. and Russell, S. E. (2001) Genomic organization, complex splicing pattern and expression of a human septin gene on chromosome 17q25.3. Oncogene 20, 5930-5939.

Mino, A., Tanaka, K., Kamei, T., Umikawa, M., Fujiwara, T. and Takai, Y. (1998) Shs1p: a novel member of septin that interacts with spa2p, involved in polarized growth in saccharomyces cerevisiae. Biochem. Biophys. Res. Commun. 251, $732-736$

Nagata, K., Asano, T., Nozawa, Y. and Inagaki, M. (2004) Biochemical and cell biological analyses of a mammalian septin complex, Sept7/9b/11. J. Biol. Chem. 279, 55895-55904.

Russell, S. E. and Hall, P. A. (2005) Do septins have a role in cancer? Br. J. Cancer 93, 499-503.

Sheffield, P. J., Oliver, C. J., Kremer, B. E., Sheng, S., Shao, Z. and Macara, I. G. (2003) Borg/septin interactions and the assembly of mammalian septin heterodimers, trimers, and filaments. J. Biol. Chem. 278, 3483-3488.

Surka, M. C., Tsang, C. W. and Trimble, W. S. (2002) The mammalian septin MSF localizes with microtubules and is required for completion of cytokinesis. Mol. Biol. Cell 13, 35323545.

Versele, M., Gullbrand, B., Shulewitz, M. J., Cid, V. J., Bahmanyar, S., Chen, R. E., Barth, P., Alber, T. and Thorner, J. (2004) Proteinprotein interactions governing septin heteropentamer assembly and septin filament organization in Saccharomyces cerevisiae. Mol. Biol. Cell 15, 4568-4583.

Versele, M. and Thorner, J. (2005) Some assembly required: yeast septins provide the instruction manual. Trends Cell Biol. 15, 414424. 